\title{
More about peer review: is it time for double-blind reviews?
}

\section{JOHN R. KLEIN}

University of Texas Health Science Center at Houston, Department of Basic Sciences, Dental Branch, Houston,TX 77030, USA. (John.R.Klein@ uth.tmc.edu)

The article by Benedita Rocha, "Trouble with peer review", was insightful and timely, and the causes of current problems with the review process were delineated well. They stem from many factors, including the ever-increasing scope and pace of science as it is practiced today, and are likely to continue into the future. Rocha provides seven good suggestions for improving the review process. I would like to add an eighth possibility to that list: the opportunity for investigators to have their work evaluated anonymously. In such a system, the journal, as well as its editorial board and staff, would have details of the authors and their affiliated institutions (all submissions would require at least one copy of the manu- script with this information provided in a standard fashion). However, at the author's request, reviewers would receive manuscript copies with this information omitted.

How would this system benefit the review process? First, it would more rigorously focus the review on scientific content and, therefore, limit the tendency of reviewers to consider subjective factors such as recognition of the author names and/or affiliations. From a purely scientific perspective, there is no need for reviewers to know the source of the work they are evaluating; the high or low quality of the work will be evident regardless of any extraneous information pertaining to authorship or affiliation. Indeed, the quality of the data should be easier to judge when viewed strictly as scientific information. Similarly, in such a system, reviewers should be prone to maintaining high quality critiques because they will have little or no clear knowledge of the origins of the work under review. Secondly, even when attempting to keep reviews on an even ethical plane, political issues frequently enter the process. With a lack of information about the source, reviewers of anonymous work will be less likely to generate overly generous or overly critical summaries. Finally, if authors know their work has been assessed based on its scientific content alone, they are less likely to feel that biases have occurred during the review process.

Clearly, given the choice, not every individual will opt for a system of anonymous review. In addition, under certain circumstances, anonymous review may run into difficulties. It is not uncommon, for example, for investigators to cite and discuss their previous work as part of the background information provided in the introduction of a manuscript. However, individuals wishing to maintain anonymity during the review process could cite such studies in the third person, which is by no means an unreasonable alternative and one that not long ago was considered to be the correct protocol for reporting scientific information.

The extent to which "double-blind" reviews will improve the manuscript review process remains to be determined. But it may help considerably, and it's worth a try.

1. Rocha, B. Nature Immunol. 2, 277 (2001) 